\title{
A STEFAN PROBLEM FOR A REACTION-DIFFUSION SYSTEM
}

\author{
AVNER FRIEDMAN*, DAVID S. ROSS ${ }^{\dagger}$ AND JIANHUA ZHANG ${ }^{\ddagger}$
}

\begin{abstract}
The paper deals with a Stefan problem for a system of three weakly coupled semilinear parabolic equations. The system describes dissolution of a spherical particle in solution. The dissolved species $A$ reacts chemically with species $B$ already in the solution, thereby forming species $C$. Species $C$ diffuses in the solution and some of it adsorbs to the particle's boundary and gradually shuts down the dissolution. It is shown that the mathematical model has a unique solution with finite shut-down time. When the reaction rate $K$ increases to infinity, the limit model should exhibit phase separation between $A$ and $B$ and it thus has two free boundaries: the particle's boundary, and the $A-B$ interface. It is proved, in the case in which $A$ and $B$ diffuse at the same rate, that the solution with finite $K$ converges to the solution of the limit problem, and that the $A$ phase in the limit problem disappears in finite time.
\end{abstract}

$\S 1$. The model. Consider a solid spherical particle composed of chemical $A$ with uniform concentration $A^{*}$. The particle is in a solution of chemical $B$. As the particle dissolves, the $A$ that enters the solution reacts with $B$ to form chemical $C$. Then $C$ diffuses in the solution and some of it reaches the solid particle and adsorbs to its surface. The presence of the adsorbed $C$ inhibits the dissolution, and ultimately shuts it down entirely.

Assuming radially symmetric data and radially symmetric functions $A, B, C$, we denote by $r=R(t)$ the radius of the solid sphere at time $t$. Then the equations

$$
\begin{aligned}
& \frac{\partial A}{\partial t}=D_{A} \Delta A-K A B, \\
& \frac{\partial B}{\partial t}=D_{B} \Delta B-K A B, \\
& \frac{\partial C}{\partial t}=D_{C} \Delta C+K A B,
\end{aligned}
$$

hold in $\{r>R(t)\}$, where $K$ is the reaction rate and $D_{A}, D_{B}, D_{C}$ are the diffusion coefficients. These equations indicate that $A$ and $B$ are lost in a second-order reaction in which $C$ is formed, and all three species diffuse. In the standard mass-action model of chemical kinetics, the concentrations are all expressed in moles/liter, and the coefficient $K$, the second-order reaction rate, is expressed in liters/(mole-sec). Then $K A B$ is the number of moles per liter per second that undergo reaction; in our case, $A$ and $B$ are consumed, $C$ is created, the same number of moles of $A$ and $B$ are lost, and this number of moles of $C$ is created. A nice reference for this material is the book by Erdi and Toth [4].

Next,

$$
\frac{d R}{d t}=\alpha \frac{\partial A}{\partial r} \quad \text { on } \quad r=R(t)
$$

where $\alpha$ is a positive constant, i.e., the rate at which the radius of the particle decreases is proportional to the flux of species $A$ away from the particle. We also have

$$
\frac{\partial B}{\partial r}=0 \quad \text { on } \quad r=R(t)
$$

\footnotetext{
* Institute for Mathematics and its Applications, University of Minnesota, Minneapolis, Minnesota 55455,

$\dagger$ Applied Mathematics and Statistics Group, Computational Science Laboratory, Eastman Kodak Company, Rochester, New York 14650-2205

$\ddagger$ University of Minnesota, School of Mathematics, Minneapolis, Minnesota 55455
} 
i.e., there is no flux of $B$ through the particle's surface and $B$ does not undergo any surface reaction.

The adsorption of $C$ to the surface is proportional to the local saturation; it is given by an empirical law $D_{C} \partial C / \partial r=\gamma C^{n}$ for some positive constants $\gamma, n$ (see [13, pp. 104-105]); for definiteness we take $n=4$, that is,

$$
D_{C} \frac{\partial C}{\partial r}=\gamma C^{4}
$$

However, all the results of this paper remain valid with minor changes if we replace $\gamma C^{4}$ by any other monotone increasing function $f(C)$ with $f(0)=0, f(C)>0$ for $C>0$.

The boundary conditions at $r=\infty$ are

$$
A(\infty, t)=0, B(\infty, t)=B^{*}, \quad C(\infty, t)=0
$$

where $B^{*}$ is a positive constant.

We now impose initial conditions. First,

$$
R(0)=R_{0}>0
$$

Next we assume that

$$
A(r, 0)=A_{0}(r), B(r, 0)=B_{0}(r), C(r, 0)=C_{0}(r)
$$

for $r>R_{0}$ where $A_{0}, B_{0}, C_{0}$ are approximately $0, B^{*}, 0$ (i.e., initially mostly only $B$ is present in the solution and its concentration is nearly uniform). We also assume that the initial conditions are smooth and fit smoothly with the boundary conditions:

$$
\begin{aligned}
& \left\{\begin{array}{l}
A_{0} \in C^{2+\nu}\left[R_{0}, \infty\right), A_{0}^{\prime}(r) \leq 0, A_{0}\left(R_{0}\right)=A^{*}, \\
A_{0}(r)=0 \text { if } r>R_{0}+\delta_{1} \text { for some } \delta_{1}>0
\end{array}\right. \\
& \left\{\begin{array}{l}
B_{0} \in C^{2+\nu}\left[R_{0}, \infty\right), B_{0}(r) \geq 0, B_{0}^{\prime}(r) \geq 0, \\
B_{0}(r)=B^{*} \text { if } r \geq R_{0}+\delta_{2} \text { for some } \delta_{2}>0 ;
\end{array}\right. \\
& \left\{\begin{array}{l}
C_{0}(r) \in C^{2}\left[R_{0}, \infty\right), C_{0}(r) \geq 0, \\
C_{0}(r)=0 \text { if } r>R_{0}+\delta_{3} \text { for some } \delta_{3}>0, \\
D_{C} \frac{\partial C_{0}}{\partial r}=\gamma C_{0}^{4} \text { at } r=R_{0},
\end{array}\right.
\end{aligned}
$$

for some $0<\nu<1$.

We finally need to determine the boundary condition for $A$ at the particle's surface. The flux of $A$ from the particle depends on the amount of $C$ that is adsorbed to the surface. On a portion of $\{r=R\}$ where there is no adsorbed $C, A=A^{*}$, the saturation concentration of $A$; local thermodynamic equilibrium is established instantaneously. On portion which is 
fully covered by $C, \partial A / \partial r=0$, i.e., the dissolution shuts down. This is actually a microscopic statement, which we shall now "average." We shall use the "weighted average"

$$
\zeta(t)=\frac{\left.\beta \int_{0}^{t} R^{2}(s) D_{C} \frac{\partial C}{\partial r}\right|_{r=R(s)} d s+\delta}{R^{2}(t)}
$$

where $\beta$ is a positive empirical parameter, and $\delta$ is a small positive parameter such that $\delta / R_{0}^{2}<$ 1. We then impose the boundary condition

$$
-\zeta_{0}(t) D_{A} \frac{\partial A}{\partial r}+\left(1-\zeta_{0}(t)\right)\left(A-A^{*}\right)=0 \quad \text { on } \quad r=R(t)
$$

where

$$
\zeta_{0}(t)=\min \{\zeta(t), 1\}
$$

Thus the dissolution shuts down as soon as $\zeta(t)$ becomes equal to 1 . This boundary condition has the basic properties demanded by the physical problem; it reduces to the Dirichlet condition in the absence of adsorbed $C$, it reduces to the Neumann condition when the surface is covered, and it makes a continuous monotone transition between these two conditions as a function of the fraction of surface area that is covered.

REMARK 1.1. The parameter $\delta$ in (1.13) ensures that $\zeta(0)>0$ and therefore the boundary condition in (1.14) does not degenerate at $t=0$. All the results of this paper, however, except uniqueness, extend to the case $\delta=0$ by simply going to the limit with $\delta \rightarrow 0$. If $\delta=0$, the solution is not smooth at $\left(R_{0}, 0\right)$ and our proof of uniqueness (for the case $\delta>0$ ) does not carry through.

For additional information on the model see [9; Chap. 18].

\section{$\S 2$. The main results.}

Definition 2.1. We refer to the system (1.1)-(1.15) as Problem $(P)$. By a solution to Problem $(P)$ we mean $(A, B, C, R)$ satisfying $(1.1)-(1.15)$ in the classical case; in particular, $R(t)$ is continuously differentiable for all $t \geq 0$.

DEFINITION 2.2. Suppose $T^{*}$ is such that

$$
\zeta(t)<1 \quad \text { if } \quad t<T^{*}, \quad \zeta(t) \geq 1 \quad \text { if } \quad t \geq T^{*} .
$$

Then we call $T^{*}$ the shut-down time.

Note that (1.4) and (1.14) reduce to

$$
R(t)=R\left(T^{*}\right), A_{r}\left(R\left(T^{*}\right), t\right)=0 \text { for } t>T^{*} .
$$

THEOREM 2.1. There exists a unique solution of $(P)$, and it has the following properties: (i) $R(t)>0, R^{\prime}(t) \leq 0$ and $\zeta^{\prime}(t)>0$ for all $t>0$; (ii) it has a finite shut-down time $T^{*}$ and $R^{\prime}(t)<0$ if $0<t<T^{*}$, and (iii) $R$ and $\zeta$ belong to $C^{1+\mu}[0, \infty) \cap C^{\infty}\left(0, T^{*}\right)$ for any $0<\mu<1$.

We are interested in the case of fast reaction, that is, $K \gg 1$. This motivates the study of the solution

$$
\left(A_{K}, B_{K}, C_{K}, R_{K}\right) \text { of }(P)
$$


as $K \rightarrow \infty$. It can be shown that

$$
\int_{0}^{T} \int_{R_{K}(t)}^{\infty} A_{K} B_{K} \leq \frac{\text { const. }}{K},
$$

and that

$$
\frac{\partial}{\partial r} A_{K} \leq 0, \frac{\partial}{\partial r} B_{K} \geq 0 .
$$

It follows, formally, that the limits

$$
\begin{aligned}
& A=\lim A_{K}, B=\lim B_{K}, C=\lim C_{K}, \\
& R=\lim R_{K} \quad \text { and } \quad \zeta=\lim \zeta_{K}
\end{aligned}
$$

are such that $A B=0$, i.e.,

$$
\begin{aligned}
A(r, t)>0 & \text { if } \quad R(t)<r<S(t), \\
& =0 \quad \text { if } \quad r>S(t), \\
B(r, t)>0 & \text { if } \quad r>S(t) \\
= & 0 \text { if } \quad R(t)<r<S(t)
\end{aligned}
$$

for some function $S(t)$; furthermore,

$$
\begin{gathered}
\frac{\partial A}{\partial t}=D_{A} \Delta A \quad \text { if } \quad R(t)<r<S(t), \quad t>0, \\
\frac{\partial B}{\partial t}=D_{B} \Delta B \quad \text { if } \quad S(t)<r<\infty, \quad t>0, \\
\frac{\partial C}{\partial t}=D_{C} \Delta C-\left(\left.D_{A} \frac{\partial A}{\partial r}\right|_{r=S(t)}\right) \delta(r-S(t)) \quad \text { if } \quad R(t)<r<\infty, t>0, \\
\frac{d R}{d t}=\left.\alpha \frac{\partial A}{\partial r}\right|_{r=R(t)}, \\
-\zeta_{0} D_{A} \frac{\partial A}{\partial r}+\left(1-\zeta_{0}\right)\left(A-A^{*}\right)=0 \quad \text { at } \quad r=R(t)
\end{gathered}
$$

where $\zeta_{0}$ is defined by (1.13), (1.15),

$$
\begin{aligned}
& D_{C} \frac{\partial C}{\partial r}=\gamma C^{4} \text { on } r=R(t), \\
& A(S(t), t)=0, \\
& B(S(t), t)=0,
\end{aligned}
$$




$$
D_{A} \frac{\partial A}{\partial r}=-D_{B} \frac{\partial B}{\partial r} \quad \text { on } \quad r=S(t) ;
$$

since $A+B \rightarrow C$, the generation of $C$ which occurs at $r=S(t)$ is at the same rate as $D_{A} \partial A / \partial r$, or $-D_{B} \partial B / \partial r$, which explains both (2.13) and the source term in (2.7).

We finally have the initial conditions:

$$
\begin{array}{lll}
A(r, 0)=A_{0}(r) & \text { if } \quad & R(0)<r<S(0), \\
B(r, 0)=B_{0}(r) & \text { if } \quad & S(0)<r<\infty, \\
C(r, 0)=C_{0}(r) & \text { if } & R(0)<r<\infty,
\end{array}
$$

( $r_{0}$ is uniquely determined), and the conditions at $r=\infty$ :

$$
B(\infty, t)=B^{*}, C(\infty, t)=0 .
$$

Definition 2.3. We shall denote the problem $(P)$ by $\left(P_{K}\right)$ and refer to the system $(2.5)-(2.16)$ as Problem $\left(P_{\infty}\right)$. By a solution to Problem $\left(P_{\infty}\right)$ we mean $\left(A, B, C, R, S, T_{f}\right)$ such that all the equations are satisfied in the classical case for $0<t<T_{f}$, and

$$
\begin{gathered}
R(t)<S(t)<\infty \quad \text { if } \quad t<T_{f}, \\
S(t)-R(t) \rightarrow 0 \quad \text { if } \quad t \rightarrow T_{f} ;
\end{gathered}
$$

in particular, $R(t)$ and $S(t)$ are continuously differentiable for $0<t<T_{f}$, and continuous for $0 \leq t \leq T_{f}$, and $A_{r}(r, t)$ is continuous for $R(t) \leq r \leq S(t)$.

The curve $r=S(t)$ is the interface between the separated phases $A$ and $B$, and $T_{f}$ is the final time, i.e., the time at which phase $A$ has totally disappeared.

Theorem 2.2. Assume that $D_{A}=D_{B}$ and $C_{0}(r) \not \equiv 0$. Then there exists a unique solution to Problem $\left(P_{\infty}\right)$, and it has the following properties: (i) $R(t)>0, R^{\prime}(t) \leq 0$ and $\zeta^{\prime}(t)>0$ for all $0<t<T_{f}$, and $R^{\prime}(t)<0$ as long as $\zeta(t)<1$; (ii) $T_{f}<\infty$, and (iii) $R, S$ and $\zeta$ belong to $C^{0}\left[0, T_{f}\right] \cap C^{1+\mu}\left[0, T_{f}\right) \cap C^{\infty}\left(0, T_{f}\right)$.

Theorem 2.3. Assume that $D_{A}=D_{B}$. Then, as $K \rightarrow \infty$, the limits in (2.2) exist where ( $\left.A, B, C, R, S, T_{f}\right)$ is the solution to Problem $\left(P_{\infty}\right)$; the convergence of $A_{K}, B_{K}, C_{K}$ is uniform in any compact subset of

$$
\left\{R(t)<r<S(t), 0 \leq t<T_{f}\right\} \cup\left\{S(t)<r<\infty, 0 \leq t<T_{f}\right\},
$$

and the convergence of $R_{K}$ and $\zeta_{K}$ is uniform for $0 \leq t<T_{f}$.

Theorem 2.1 is proved in Sections 3-6, and Theorems 2.2, 2.3 are proved in Sections 7-10.

REMARK 1. Reaction-diffusion systems of the form

$$
A_{t}=D_{A} \Delta A-K A B, \quad B_{t}=D_{B} \Delta B-K A B
$$


with $K \rightarrow \infty$ have been studied in [1] [12]. Evans [5] considered (2.18) in a fixed cylinder $\Omega \times(0, T)$ under the assumptions

$$
\frac{\partial A}{\partial n}=0 \quad \text { on } \quad \partial \Omega, \quad \frac{\partial B}{\partial n}=0 \text { on } \partial \Omega, \quad A_{0} B_{0}=0
$$

where $A_{0}, B_{0}$ are the nonnegative initial values for $A$ and $B$ respectively. He proved that, as $K \rightarrow \infty, A \rightarrow u^{+}$and $B \rightarrow u^{-}$where $u$ is the solution of

$$
u_{t}=\operatorname{div}(a(u) \nabla u)=0 \quad \text { in } \quad \Omega \times(0, T)
$$

with $\partial u / \partial n=0$ on $\partial \Omega$ and $\left.u\right|_{t=0}=A_{0}-B_{0}$; here

$$
a(u)=D_{A} \quad \text { if } \quad u>0, a(u)=D_{B} \quad \text { if } \quad u<0 .
$$

(Uniqueness for the limit problem was established in [2].) His proof relies heavily on the assumptions in (2.19) and does not seem to extend to the present case where we have a moving boundary $r=R(t)$ and different boundary conditions than in (2.19).

REMARK 2. For the general study of the Stefan problem in $n$-dimensions we refer to [8] [10] and the references therein. In the case of radially symmetric solutions, existence, uniqueness and regularity have been established by several methods (see [6] [7] and the references therein). In the standard Stefan problem one assumes that $A$ vanishes on the free boundary. The condition (1.14) is called a "kinetic" condition. A Stefan problem for one heat equation in one-dimension with kinetic condition, was studied by Visintin [14] and Xie [15].

$\S 3$. Local existence for (P). In this section we prove:

Theorem 3.1. There exists a solution $(A, B, C, R)$ of Problem $(P)$ for $0<t<T$, where $T$ is some small positive number.

Proof. The proof is based on a fixed-point argument. Set, for any $N_{0}>0$,

$$
\begin{aligned}
& K_{R}=\left\{R(t) \in C^{0,1}[0, T], R(0)=R_{0},-M \leq \dot{R}(t) \leq 0 \text { a.e. }\right\}, \\
& K_{\zeta}=\left\{\zeta(t) \in C^{0,1}[0, T], \frac{\delta}{R_{0}^{2}} \leq \zeta(t) \leq N_{0}, 0 \leq \dot{\zeta}(t) \leq N \text { a.e. }\right\}
\end{aligned}
$$

where

$$
M=\frac{\alpha}{D_{A}} \frac{A^{*} R_{0}^{2}}{\delta}
$$

and $N$ is a positive constant to be determined. We endow $K_{R}$ and $K_{\zeta}$ with the $C^{0}[0, T]$-norm; then $K_{R} \times K_{\zeta}$ is a compact set in $C^{0}[0, T] \times C^{0}[0, T]$.

For each $(R(t), \zeta(t)) \in K_{R} \times K_{\zeta}$ there exists a unique solution $(A(r, t), B(r, t))$ of $(1.1)$, $(1.2),(1.5),(1.14),(1.7)$ with the initial conditions as in (1.9); since the parabolic system is weakly coupled, such a solution exists for any given time $T$. By the maximum principle,

$$
0 \leq A(r, t) \leq A^{*}, 0 \leq B(r, t) \leq B^{*} .
$$

We next prove that

$$
A_{r}(r, t) \leq 0, B_{r}(r, t) \leq 0 .
$$


If we differentiate (1.1), (1.2) with respect to $r$, we get a coupled system of parabolic equations

$$
\begin{aligned}
\frac{\partial}{\partial t} A_{r}-\mathcal{L} A_{r} & =-K A B_{r} \\
\frac{\partial}{\partial t} B_{r}-\mathcal{L} B_{r} & =-K B A_{r}
\end{aligned}
$$

where $\mathcal{L}$ is an elliptic operator. On $t=0$ and on the boundary $r=R(t)$ we have $A_{r} \leq 0, B_{r} \geq 0$. We approximate $A_{r}, B_{r}$ by solutions $A_{r}^{\varepsilon}, B_{r}^{\varepsilon}$ satisfying the same parabolic system, with initial and boundary conditions given by

$$
A_{r}^{\varepsilon}=A_{r}-\varepsilon, B_{r}^{\varepsilon}=B_{r}+\varepsilon .
$$

Then $A_{r}^{\varepsilon}<0, B_{r}^{\varepsilon}>0$ for $R(t) \leq r<\infty, 0 \leq t \leq T$. Indeed, otherwise there is a smallest $t_{0}$ such that $A_{r}^{\varepsilon} \leq 0, B_{r}^{\varepsilon} \geq 0$ for $R(t) \leq r<\infty, 0 \leq t \leq t_{0}$, and $A_{r}^{\varepsilon}=0$ or $B_{r}^{\varepsilon}=0$ at some point $\left(r_{0}, t_{0}\right)$. This is a contradiction to the strong maximum principle applied to $A_{r}^{\varepsilon}$ or to $B_{r}^{\varepsilon}$.

If we now let $\varepsilon \rightarrow 0$, we obtain the assertion (3.2).

Motivated by (1.4), (1.14), we now define

$$
\bar{R}(t)=R_{0}+\frac{\alpha}{D_{A}} \int_{0}^{t} \frac{1-\zeta_{0}(s)}{\zeta_{0}(s)}\left[A(R(s), s)-A^{*}\right] d s .
$$

Next we consider the parabolic equation (1.3) in $\bar{R}(t)<r<\infty, 0<t<T$ with boundary conditions (1.6) and $C(\infty, t)=0$, and with initial conditions $C(r, 0)=C_{0}(r)$. Since the functions $K A^{*} B^{*} t+\hat{C} \quad\left(\widehat{C}=\sup C_{0}\right)$ and 0 are supersolution and sub-solution, respectively, the existence of a solution can be established by a fixed-point argument (cf. [6; Chap. 7, Sec. 5]). Uniqueness follows by a comparison principle [6].

We now define

$$
\bar{\zeta}(t)=\frac{\beta \gamma \int_{0}^{t} \bar{R}^{2}(s) C^{4}(\bar{R}(s), s)+\delta}{\bar{R}^{2}(t)}
$$

and consider the mapping $W$ :

$$
W(R(t), \zeta(t))=(\bar{R}(t), \bar{\zeta}(t))
$$

If we show that $W$ has a fixed point in $K_{R} \times K_{\zeta}$, then this yields a solution to Problem $(P)$.

LEMMA 3.2. $W$ maps $K_{R} \times K_{\zeta}$ into itself.

Proof. From (3.3), (3.4) we get

$$
\begin{aligned}
& \frac{d}{d t} \bar{R}(t)=\frac{\alpha}{D_{A}} \frac{1-\zeta_{0}(t)}{\zeta_{0}(t)}\left(A-A^{*}\right), \\
& \frac{d}{d t} \bar{\zeta}(t)=\beta \gamma C^{4}(\bar{R}(t), t)-\frac{2}{\bar{R}(t)} \bar{\zeta}(t) \frac{d \bar{R}(t)}{d t} .
\end{aligned}
$$


From (3.5) we see that

$$
-M \leq \frac{d \bar{R}(t)}{d t} \leq 0
$$

Since $C \leq K A^{*} B^{*} t+\widehat{C}$ we find from (3.4) that $\bar{\zeta}(t) \leq N_{0}$ if $T$ is small enough. From (3.6) and (3.7) it then follow that

$$
0<\frac{d}{d t} \bar{\zeta}(t) \leq \widehat{N}
$$

provided $\widehat{N}$ is a sufficiently large number independent of $N$ (see the definition of $K_{\zeta}$ ). Hence, choosing $N=\widehat{N}$ we see that $(\bar{R}, \bar{\zeta})$ belongs to $K_{R} \times K_{\zeta}$.

LEMma 3.3. $W$ is continuous (when $K_{R} \times K_{\zeta}$ is endowed with the $C^{0}[0, T] \times C^{0}[0, T]$ norm).

Proof. If we use the transformation $\hat{r}=r-R(t)$ in order to flatten the boundary $r=R(t)$, we get a new parabolic equation for $A$ where a new term $\dot{R} A_{r}$ is added to the heat operator. On the lateral boundary $\widehat{r}=0$

$$
-A_{r}+a(t) A=b(t)
$$

where $a, b$ are Lipschitz continuous and their Lipschitz constants are bounded independently of $(R, \zeta)$ in $K_{R} \times K_{\zeta}$. We can therefore apply $L^{p}$-estimates [10] to deduce that

$$
\left\|A_{r}\right\|_{L^{p}\left(\Omega_{T}\right)}+\left\|A_{r r}\right\|_{L^{p}\left(\Omega_{T}\right)}+\left\|A_{t}\right\|_{L^{p}\left(\Omega_{T}\right)} \leq C_{1}
$$

for any $1<p<\infty$, where

$$
\Omega_{T}=\{R(t) \leq r<\infty, 0 \leq t \leq T\}
$$

and $C_{1}$ is a constant depending on $p$ but not on $(R, \zeta)$ and $T$. By Sobolev's imbedding [11] we then have the Hölder estimate

$$
\left\|A_{r}\right\|_{C^{\mu}\left(\Omega_{T}\right)} \leq C_{2}
$$

for some $0<\mu<1$ and $C_{2}$ independent of $T$ and $(R, \zeta)$.

We now proceed to prove that $W$ is continuous.

Suppose $\left(R_{n}, \zeta_{n}\right)$ and $(R, \zeta)$ belong to $K_{R} \times K_{\zeta}$ and $R_{n} \rightarrow R, \zeta_{n} \rightarrow \zeta$ in the $C^{0}[0, T]$-norm. We need to prove that

$$
W\left(R_{n}, \zeta_{n}\right) \rightarrow W(R, \zeta)
$$

Define $A_{n}, B_{n}, \bar{\zeta}_{n}, \bar{R}_{n}$ and $C_{n}$ corresponding to $R_{n}, \zeta_{n}$, so that $W\left(R_{n}, \zeta_{n}\right)=\left(\bar{R}_{n}, \bar{\zeta}_{n}\right)$. Applying the estimates (3.8), (3.9) to $A_{n}$ and similar estimates to $B_{n}$ and $C_{n}$, we can easily show that any subsequence of $n$ 's has a subsequence for which

$$
A_{n} \rightarrow \tilde{A}, B_{n} \rightarrow \tilde{B}
$$


where $\tilde{A}, \widetilde{B}$ satisfy the same parabolic system in $R(t)<r<\infty, 0<t<T$ which $A, B$ satisfy. By uniqueness it follows that $\tilde{A}=A$ and $\widetilde{B}=B$. Therefore

$$
A_{n} \rightarrow A, B_{n} \rightarrow B \text { uniformly in } \rho_{n}(t) \leq r<\infty, 0 \leq t \leq T
$$

where $\rho_{n}(t)=\max \left\{R_{n}(t), R(t)\right\}$.

We proceed to prove that

$$
\bar{R}_{n}(t)-\bar{R}(t)=\frac{\alpha}{D_{A}}\left\{\int_{0}^{t} \frac{1-\zeta_{n, 0}(s)}{\zeta_{n, 0}(s)}\left[A_{n}\left(R_{n}(s), s\right)-A^{*}\right] d s\right.
$$

$$
\left.-\int_{0}^{t} \frac{1-\zeta_{0}(s)}{\zeta_{0}(s)}\left[A(R(s), s)-A^{*}\right] d s\right\} \rightarrow 0
$$

where $\zeta_{n, 0}=\min \left\{\zeta_{n}, 1\right\}$. By $(3.9)$

$$
\begin{aligned}
& \left|A_{n}\left(R_{n}(s), s\right)-A_{n}\left(\rho_{n}(s), s\right)\right| \leq C_{1}\left|R_{n}(s)-R(s)\right|^{\mu}, \\
& \left|A(R(s), s)-A\left(\rho_{n}(s), s\right)\right| \leq C_{1}\left|R_{n}(s)-R(s)\right|^{\mu} .
\end{aligned}
$$

Using also (3.10) we conclude that

$$
\left|\frac{\alpha}{D_{A}} \int_{0}^{t} \frac{1-\zeta_{n, 0}(s)}{\zeta_{n, 0}(s)}\left[A_{n}\left(R_{n}(s), s\right)-A(R(s), s)\right] d s\right| \leq C_{2} \int_{0}^{t}\left|R_{n}(s)-R(s)\right|^{\mu} d s+\varepsilon_{n}
$$

where $\varepsilon_{n} \rightarrow 0$ uniformly in $t$, as $n \rightarrow \infty$. Since $\zeta_{n}(t) \rightarrow \zeta(t)$ uniformly, we deduce from the expression for $\bar{R}_{n}-\bar{R}$ in (3.11) that

$$
\left|\bar{R}_{n}(t)-\bar{R}(t)\right| \rightarrow 0 \quad \text { uniformly in } t \in[0, T] .
$$

Similarly we can prove that $C_{n} \rightarrow C$ for $\bar{R}(t)<r<\infty, 0 \leq t \leq T$ and $\bar{\zeta}_{n}(t) \rightarrow \bar{\zeta}(t)$ uniformly in $0 \leq t \leq T$, and this completes the proof of Lemma 3.3 .

From Lemmas 3.2, 3.3 we have that $W$ maps the compact set $K_{R} \times K_{\zeta}$ into itself and is continuous. By the Schauder fixed-point theorem, $W$ has a fixed point $(R, \zeta)$ and this completes the proof of Theorem 3.1 .

§. Uniqueness. In this section we prove:

Theorem 4.1. For any $T>0$, Problem $(P)$ has at most one solution.

Proof. Suppose there are two solutions $\left(A_{i}, B_{i}, C_{i}, R_{i}, \zeta_{i}\right) \quad(i=1,2)$. Set

$$
\hat{A}_{i}(x, t)=A_{i}(r, t), \hat{B}_{i}(x, t)=B_{i}(r, t), \hat{C}_{i}(x, t)=C_{i}(r, t)
$$

where $x=r-R_{i}(t)$. Then

$$
\begin{aligned}
& \hat{A}_{i, t}=D_{A}\left(\hat{A}_{i, x x}+\frac{2}{x+R_{i}} \hat{A}_{i, x}\right)+\dot{R}_{i} \hat{A}_{i, x}-K \hat{A}_{i} \hat{B}_{i} \\
& \quad \text { in } Q_{T}=\{x>0, t>0\}, \\
& -D_{A} \zeta_{i, 0} \hat{A}_{i, x}+\left(1-\zeta_{i, 0}\right)\left(\hat{A}_{i}-A^{*}\right)=0 \text { for } x=0, t>0, \\
& \hat{A}_{i}(x, 0)=A_{0}\left(x+R_{0}\right) \text { for } x>0, \\
& \lim _{x \rightarrow \infty} \hat{A}_{i}(x, t)=0 \text { for } t>0 .
\end{aligned}
$$


Similar systems can be written for $\widehat{B}_{i}$ and $\widehat{C}_{i}$. Set

$$
u=\hat{A}_{1}-\hat{A}_{2}, v=\hat{B}_{1}-\hat{B}_{2}, w=\hat{C}_{1}-\hat{C}_{2}
$$

and

$$
\rho=R_{1}-R_{2}, \eta=\zeta_{1}-\zeta_{2}
$$

Then

$$
\dot{\rho}(t)=\frac{\alpha}{D_{A}}\left[\frac{1-\zeta_{1,0}(t)}{\zeta_{1,0}(t)}\left(\hat{A}_{1}(x, 0)-A^{*}\right)-\frac{1-\zeta_{2,0}(t)}{\zeta_{2,0}(t)}\left(\hat{A}_{2}(x, 0)-A^{*}\right)\right]
$$

so that

$$
|\dot{\rho}(t)| \leq C[|u(0, t)|+|\eta(t)|] .
$$

Next we easily estimate from (1.13),

$$
|\eta(t)| \leq C\left[\|\rho\|_{L^{\infty}(0, T)}+\|w(0, t)\|_{L^{\infty}(0, T)}\right] .
$$

Since $\rho(0)=0,(4.2)$ yields

$$
\|\rho\|_{L^{\infty}(0, T)} \leq C T\left[\|u(0, t)\|_{L^{\infty}(0, T)}+\|\eta\|_{L^{\infty}(0, T)}\right] .
$$

Substituting this into (4.3), we get

$$
\|\eta\|_{L^{\infty}(0, T)} \leq \frac{C T}{1-C T}\left[\|u(0, t)\|_{L^{\infty}(0, T)}+\|w(0, t)\|_{L^{\infty}(0, T)}\right]
$$

if $T$ is such that $C T<1$. Finally, using (4.5) in (4.4) we get

$$
\|\rho\|_{L^{\infty}(0, T)} \leq C T\left[\|u(0, t)\|_{L^{\infty}(0, T)}+\|w(0, t)\|_{L^{\infty}(0, T)}\right] .
$$

From (4.1) we see that $u$ satisfies a parabolic system

$$
\begin{aligned}
& u_{t}=D_{A} u_{x x}+\left(\frac{2 D_{A}}{x+R_{1}}+\dot{R}_{1}\right) u_{x}-K \hat{B}_{1} u+F \text { in } Q_{T}, \\
& -D_{A} \zeta_{1,0} u_{x}+\left(1-\zeta_{1,0}\right) u=G \text { if } x=0, t>0, \\
& u(x, 0)=0, x>0 \\
& \lim _{x \rightarrow \infty} u(x, t)=0, t>0 .
\end{aligned}
$$

where

$$
\begin{aligned}
|F| & \leq C\left[\|\dot{\rho}\|_{L^{\infty}(0, T)}+\|v\|_{L^{\infty}\left(Q_{T}\right)}\right] \\
& \leq C\left[\|u\|_{L^{\infty}\left(Q_{T}\right)}+\|v\|_{L^{\infty}\left(Q_{T}\right)}+\|w\|_{L^{\infty}\left(Q_{T}\right)}\right]
\end{aligned}
$$

by (4.2), (4.5), and

$$
|G| \leq C|\eta(t)| \leq C T\left[\|u\|_{L^{\infty}\left(Q_{T}\right)}+\|w\|_{L^{\infty}\left(Q_{T}\right)}\right] .
$$


It is easy to see that the function

$$
C(t+T) \cdot\left[\|u\|_{L^{\infty}\left(Q_{T}\right)}+\|v\|_{L^{\infty}\left(Q_{T}\right)}+\|w\|_{L^{\infty}\left(Q_{T}\right)}\right]
$$

is a supersolution to (4.7), so that

$$
\|u\|_{L^{\infty}\left(Q_{T}\right)} \leq C T\left[\|u\|_{L^{\infty}\left(Q_{T}\right)}+\|v\|_{L^{\infty}\left(Q_{T}\right)}+\|w\|_{L^{\infty}\left(Q_{T}\right)}\right] .
$$

The same estimate can similarly be established for $v$ and for $w$. Hence if $T$ is small enough then $u=v=w=0$ in $Q_{T}$. We can now proceed step-by-step to prove that $u=v=w=0$ for all $t$, as long as the two solutions exist.

$\S 5$. Global existence. In this section we prove that there exists a solution to Problem $(P)$ for all time. We first recall that as long as $\zeta(t) \leq 1$

$$
\begin{gathered}
\dot{R}(t)=\frac{\alpha}{D_{A}} \frac{1-\zeta(t)}{\zeta(t)}\left(A-A^{*}\right), \\
\dot{\zeta}(t)=\beta \gamma C^{4}(R(t), t)-\frac{2 \zeta(t)}{R(t)} \dot{R}(t) .
\end{gathered}
$$

Hence

$$
\dot{R}(t)<0, \dot{\zeta}(t)>0
$$

LEMMA 5.1. There exists a positive constant $R_{*}$ such that

$$
R(t) \geq R_{*} \quad \text { as long as } \quad \zeta(t) \leq 1 .
$$

Proof. Take $\lambda$ and $\varepsilon_{1}$ positive and small such that

$$
C^{4}(R(t), t) \leq \varepsilon_{1} \quad \text { and } \quad \frac{1}{2} R_{0}<R(t)
$$

for all $\lambda \leq t \leq 2 \lambda$. For $t>2 \lambda$

$$
\zeta(t) \geq \frac{\beta \gamma \varepsilon_{1} \lambda+\delta}{R^{2}(t)}
$$

Hence $R(t)>R_{\star}$ as long as $\zeta(t) \leq 1$, where $R_{\star}=\left(\beta \gamma \varepsilon_{1} \lambda\right)^{1 / 2}$.

REMARK 5.1. Note that the lower bound $R_{*}$ is independent of the regularizing parameter $\delta$; cf. Remark 1.1.

ThEOREM 5.2. There exists a global solution to Problem $(P)$, and $R \in C^{1+\mu}[0, \infty) \cap$ $C^{\infty}\left(0, T^{*}\right)$, for any $0<\mu<1$.

Proof. Suppose we already have a solution for $0 \leq t \leq T$ where $T$ is a positive number, not necessarily small. By Lemma $5.1, R(t) \geq R_{*}$ for $0 \leq t \leq T$.

Since $R(t) \geq R_{*}>0$ ( $R_{*}$ independent of $\left.t\right)$, a review of the proof of local existence shows that the solution can be extended to $0 \leq t \leq T+T_{0}$ provided $T_{0}$ is a small positive constant depending only on an a priori bound on $\sup |\dot{R}(t)|$. By $(5.1)|\dot{R}(t)|$ is indeed uniformly bounded 
(independently on $t$ ), and therefore the solution to Problem $(P)$ can be extended step-by-step to all $t>0$.

To prove the a priori regularity of $R$ and $\zeta$, we perform a change of variables

$$
\hat{A}(x, t)=A(r, t), \hat{B}(x, t)=B(r, t), \hat{C}(x, t)=C(r, t)
$$

where $x=r-R(t)$. Then $\hat{A}$ satisfies, for any $T>0$,

$$
\begin{aligned}
& \hat{A}_{t}=D_{A}\left(\hat{A}_{x x}+\frac{2}{x+R} \hat{A}_{x}\right)+\dot{R} A_{x}-K \hat{A} \hat{B} \quad \text { in } \quad Q_{T}, \\
& -D_{A} \zeta_{0} \hat{A}_{x}+\left(1-\zeta_{0}\right)\left(\hat{A}-A^{*}\right)=0 \text { for } x=0,0<t<T, \\
& \hat{A}(x, 0)=A_{0}\left(x+R_{0}\right), x>0, \\
& \lim _{x \rightarrow \infty} \hat{A}(x, t)=0,0<t<T .
\end{aligned}
$$

Since $\dot{R}(t)$ is uniformly bounded, the same is true of $\dot{\zeta}(t)$, by $(5.2)$ (Recall that $C(R(t), t)$ is bounded by $\left.K A^{*} B^{*} T+\hat{C}\right)$. We can therefore apply the $L^{p}$ parabolic estimates [10] to $\hat{A}$ and conclude that

$$
\int_{Q_{T}}\left(\left|\hat{A}_{r}\right|+\left|\hat{A}_{r r}\right|+\left|\hat{A}_{t}\right|\right)^{p} \leq C_{p, T}
$$

for any $p>1$. This implies that

$$
\hat{A} \in C_{r, t}^{\mu, \mu / 2}\left(Q_{T}\right) \text { for } \quad 0<\mu<1,
$$

and yields the $C^{1+\mu / 2}[0, T]$ regularity of $R(t)$.

Similar $L^{p}$ estimates can be established for $\widehat{B}$ and $\widehat{C}$ and then, from (5.2), one deduces the $C^{1+\mu / 2}[0, T]$ regularity of $\zeta(t)$.

The above arguments can be used to prove step-by-step the $C^{1+\mu}[0, T]$ and $C^{\infty}(0, T)$ regularity of $R(t)$ and $\zeta(t)$ for any $0<T \leq T^{*}$

\section{$\S 6$. Finite shut-down.}

LEMMA 6.1. If $\zeta(t)<1$ for all $t>0$ then

$$
\lim _{t \rightarrow \infty} \zeta(t)=1
$$

and

$$
\lim _{t \rightarrow \infty} \dot{R}(t)=0 .
$$

Proof. The idea of the proof is to show by comparison (with linear problems) that both $A$ and $B$ do not go to zero as $t \rightarrow \infty$, and the same is then true of $C$; this implies that $\zeta(t) \rightarrow \infty$ as $t \rightarrow \infty$, which is a contradiction. Set

$$
\rho=\lim _{t \rightarrow \infty} R(t)>0, \quad \sigma=\lim _{t \rightarrow \infty} \zeta(t) .
$$


We shall assume that $\sigma<1$ and derive a contradiction. We begin by introducing the solution $\widetilde{A}, \widetilde{B}$ to

$$
\begin{aligned}
& \tilde{A}_{t}=D_{A} \Delta \tilde{A}-K B^{*} \tilde{A} \text { for } r>R(t), t>0, \\
& -D_{A} \zeta(t) \tilde{A}_{r}+(1-\zeta(t))\left(\tilde{A}-A^{*}\right)=0 \text { if } r=R(t), t>0, \\
& \tilde{A}(r, 0)=A_{0}(r) \text { if } r>R_{0}, \\
& \lim _{r \rightarrow \infty} \tilde{A}(r, t)=0 \text { for any } t>0,
\end{aligned}
$$

and

$$
\begin{aligned}
& \widetilde{B}_{t}=D_{B} \Delta \widetilde{B}-K A^{*} \widetilde{B} \text { for } r>R(t), t>0, \\
& \widetilde{B}_{r}=0 \text { if } r=R(t), t>0, \\
& \widetilde{B}(r, 0)=B_{0}(r) \text { if } r>R_{0}, \\
& \lim _{r \rightarrow \infty} \tilde{B}(r, t)=B^{*} \text { for any } t>0 .
\end{aligned}
$$

By comparison, $\widetilde{A} \leq A, \widetilde{B} \leq B$.

Let $\tilde{A}_{\infty}, \widetilde{B}_{\infty}$ denote the solutions to the limit problems $(t \rightarrow \infty)(6.3)$ and $(6.4)$ :

$$
\begin{aligned}
& D_{A} \Delta \tilde{A}_{\infty}-K B^{*} \tilde{A}_{\infty}=0, \rho<r<\infty, \\
& -D_{A} \sigma \tilde{A}_{\infty, r}+(1-\sigma)\left(\tilde{A}_{\infty}-A^{*}\right)=0, r=\rho, \\
& \lim _{r \rightarrow \infty} \tilde{A}_{\infty}(r)=0
\end{aligned}
$$

and

$$
\begin{gathered}
D_{B} \Delta \widetilde{B}_{\infty}-K A^{*} \widetilde{B}_{\infty}=0, \rho<r<\infty, \\
\widetilde{B}_{\infty, r}(\rho)=0, \\
\lim _{r \rightarrow \infty} \widetilde{B}_{\infty}(r)=B^{*} .
\end{gathered}
$$

Note that, since $\sigma<1, \tilde{A}_{\infty}(r) \geq 0$ for all $r>\rho ; \widetilde{B}_{\infty}(r)$ is positive for all $r \geq \rho$.

We shall prove that the functions

$$
\tilde{u}(r, t)=\tilde{A}(r, t)-\tilde{A}_{\infty}(r), \tilde{v}(r, t)=\tilde{B}(r, t)-\widetilde{B}_{\infty}(r)
$$

converge to zero as $t \rightarrow \infty$.

The function $\tilde{u}$ satisfies

$$
\begin{array}{r}
\tilde{u}_{t}=D_{A} \Delta \tilde{u}-K B^{*} \tilde{u} \text { if } r>R(t), t>0 \\
-D_{A} \sigma \tilde{u}_{r}+(1-\sigma) \widetilde{u}=\widetilde{\varepsilon}(t) \quad \text { if } \quad r=R(t), t>0
\end{array}
$$

where $\tilde{\varepsilon}(t) \rightarrow 0$ as $t \rightarrow \infty$. For any $\varepsilon>0$,

$$
\frac{1}{\sigma}|\widetilde{\varepsilon}(t)|<\varepsilon \quad \text { if } \quad t>t_{\varepsilon},
$$


and the function

$$
\tilde{U}(r, t)=A^{*} e^{-K B^{*}\left(t-t_{\varepsilon}\right)}+\frac{\varepsilon}{r}\left(R\left(t_{\varepsilon}\right)\right)^{2}
$$

is then a supersolution of (6.7) in $r>R(t), t>t_{\varepsilon}$, which majorizes $|\tilde{u}|$ both on $t=t_{\varepsilon}$ and as $r \rightarrow \infty$. Hence, by comparison,

$$
|\widetilde{u}(r, t)| \leq \tilde{U}(r, t)
$$

Taking $t \rightarrow \infty$ and recalling that $\varepsilon$ is arbitrary, we conclude that

$$
\sup _{r \geq R(t)}\left|\tilde{A}(r, t)-\tilde{A}_{\infty}(r)\right| \rightarrow 0 \quad \text { if } \quad t \rightarrow \infty .
$$

Similarly, the function $v$ satisfies

$$
\begin{gathered}
\tilde{v}_{t}=D_{B} \Delta \tilde{v}-K A^{*} \tilde{v} \quad \text { for } \quad r>R(t), t>0 \\
v_{r}(R(t), t) \rightarrow 0 \text { if } t \rightarrow \infty
\end{gathered}
$$

and, with a supersolution similar to (6.8), we conclude that

$$
\sup _{r \geq R(t)}\left|\widetilde{B}(r, t)-\widetilde{B}_{\infty}(r)\right| \rightarrow 0 \quad \text { if } \quad t \rightarrow \infty
$$

Since $\widetilde{A}_{\infty}(r)>0, \widetilde{B}_{\infty}(r)>0$ for $r>\rho$, it follows from $(6.9),(6.10)$ that

$$
\tilde{A}(r, t) \tilde{B}(r, t) \geq c_{0} \chi_{[a, b]} \quad \text { for } \quad t \geq t_{*}
$$

where $c_{0}$ is a positive constant, $\rho<a<b<\infty$, and $t_{*}$ is sufficiently large.

Consider the parabolic problem

$$
\begin{gathered}
\tilde{C}_{t}=D_{C} \Delta \widetilde{C}+K c_{0} \chi_{[a, b]} \text { if } r>R(t), t>t_{*}, \\
D_{C} \widetilde{C}_{r}=\gamma \widetilde{C}^{4} \quad \text { if } r=R(t), t>t_{*}, \\
\tilde{C}\left(r, t_{*}\right)=0 \text { if } r>R\left(t_{*}\right), \\
\lim _{r \rightarrow \infty} \tilde{C}(r, t)=0 \quad \text { if } t>t_{*} .
\end{gathered}
$$

Since $A B \geq \tilde{A} \widetilde{B}$, it follows from (6.11), by comparison, that

$$
C(r, t) \geq \tilde{C}(r, t) .
$$

Denote by $\tilde{C}_{\infty}$ the solution to

$$
\begin{aligned}
& D_{C} \Delta \widetilde{C}_{\infty}+K c_{0} \chi_{[a, b]}=0, r>\rho, \\
& D_{C} \widetilde{C}_{\infty, r}=\gamma \widetilde{C}_{\infty}^{4}, r=\rho, \\
& \widetilde{C}_{\infty}(\infty)=0 ;
\end{aligned}
$$


clearly $\tilde{C}_{\infty}(r)>0$ for $r \geq \rho$. We shall evaluate the difference

$$
\widetilde{w}(r, t)=\widetilde{C}(r, t)-\widetilde{C}_{\infty}(r)
$$

It satisfies

$$
\begin{aligned}
& \tilde{w}_{t}=D_{C} \Delta w \text { if } r>R(t), t>t_{*}, \\
& -D_{C} w_{r}+a(t) w=\varepsilon(t) \text { if } r=R(t), t>0, \\
& \lim _{r \rightarrow \infty} w(r, t)=0 \text { if } t>t_{*},
\end{aligned}
$$

where

$$
\varepsilon(t)=D_{C} \tilde{C}_{\infty, r}(R(t))-\gamma \tilde{C}_{\infty}^{4}(R(t)) \rightarrow 0 \quad \text { if } \quad t \rightarrow \infty
$$

and

$$
a(t)=\left(\gamma \widetilde{C}^{4}-\gamma \tilde{C}_{\infty}^{4}\right) /\left(\tilde{C}-\tilde{C}_{\infty}\right)>0
$$

As in the proof of (6.9) we now construct, for any $\varepsilon>0$, a supersolution (cf. (6.8))

$$
\widetilde{W}(r, t)=\frac{M_{\varepsilon}}{t^{3 / 2}} e^{-\frac{r^{2}}{4 t \sqrt{D_{C}}}}+\frac{\varepsilon}{r}\left(R\left(t_{\varepsilon}\right)\right)^{2} \quad \text { for } \quad t>t_{\varepsilon}
$$

where $D_{A}|\varepsilon(t)|<\varepsilon$ if $t>t_{\varepsilon}$. By comparison,

$$
|w(r, t)| \leq \widetilde{W}(r, t) \quad \text { if } \quad r>R(t), t>t_{\varepsilon} .
$$

Recalling (6.15) we conclude that

$$
\tilde{C}(R(t), t) \rightarrow \tilde{C}_{\infty}(\rho) \text { if } t \rightarrow \infty
$$

and, by (6.13),

$$
C(R(t), t) \geq \nu \quad \text { if } \quad t \geq \tilde{t} \quad\left(\nu=\frac{1}{2} \tilde{C}_{\infty}(\rho)>0\right)
$$

for some $\tilde{t}>0$.

Since $D_{C} C_{r}=\gamma C^{4}$ at $(R(t), t)$, and since $0<R_{\star} \leq R(t)<R_{0}$, we can use $(6.17)$ in (1.13) to deduce that $\zeta(t) \rightarrow \infty$ if $t \rightarrow \infty$; this is a contradiction to the assumption that $\zeta(t)<1$ for all $t$. We have thus proved that $\sigma=1$, i.e., (6.1) holds. The assertion (6.2) then follows from (5.1) and (6.1).

To prove that there is a finite shut-down time we may proceed by contradiction as before, namely, we assume that $\zeta(t)<1$ for all $t>0$, and then wish to derive a contradiction by using (1.13). This however requires a good lower bound on $C(R(t), t)$ in case $\sigma=\lim _{t \rightarrow \infty} \zeta(t)$ is equal to 1 (Note that if $\sigma=1$ then $\tilde{A}=0$ and the previous proof does not provide a useful lower bound on $C(R(t), t)$.) The desired bound is provided in the following lemma.

LEMMA 6.2. If $\zeta(t)<1$ for all $t>0$ then there exists a positive constant $M$ such that

$$
C_{r}(R(t), t) \geq \frac{M}{t^{6}}
$$


for all $t$ large.

Proof. For any $t_{0}>0, \quad C\left(r, t_{0}\right)>0$ for all $r \geq R\left(t_{0}\right)$. Choose $R\left(t_{0}\right)<a<b<\infty$ such that

$$
C\left(r, t_{0}\right)>\varepsilon_{0} \chi_{[a, b]}(r) \equiv c_{0}(r)
$$

for some $\varepsilon_{0}>0$, and define

$$
\widetilde{C}(r, t)=\int_{\mathbb{R}^{3}} \frac{e^{-\frac{|x-y|^{2}}{4 \sqrt{D_{C}}\left(t-t_{0}\right)}}}{\left(t-t_{0}\right)^{3 / 2}} \varepsilon \varepsilon_{0}(|y|) d y, \quad 0<\varepsilon<1
$$

for $r=|x|>R(t), t>t_{0}$. Since $\tilde{C}\left(R\left(t_{0}\right), t_{0}\right)=0$,

$$
C(R(t), t)>\widetilde{C}(R(t), t) \quad \text { if } \quad t_{0} \leq t \leq t_{1}
$$

for some $t_{1}>t_{0}$. On the other hand, for $r=R(t), t \geq t_{1}$ we have

$$
\begin{gathered}
-D_{C} \tilde{C}_{r}+\gamma \tilde{C}^{4} \leq-D_{C} \varepsilon \int_{\{a<|y|<b\}} \frac{|y|-R(t)}{(4 \pi)^{3 / 2} 2 \sqrt{D_{C}}\left(t-t_{0}\right)^{5 / 2}} e^{-\frac{|R(t)-y|^{2}}{4 \sqrt{D_{C}}\left(t-t_{0}\right)}} \\
+C_{1} \varepsilon^{4}<0
\end{gathered}
$$

if $\varepsilon$ is sufficiently small. Recalling also (6.19), (6.20) and noting that $\widetilde{C}$ is a subsolution of (1.3), we conclude, by comparison, that if $\varepsilon$ is small enough then

$$
C(r, t)>\widetilde{C}(r, t) \text { if } t>t_{0}
$$

from which (6.18) follows upon using (1.6).

We finally prove:

THEOREM 6.3. There is a finite shut-down time $T^{*}$.

Proof. Suppose $\zeta(t)<1$ for all $t>0$. We can write, for $r=R(t)$,

$$
\begin{aligned}
\dot{\zeta}(t) & =\beta D_{C} C_{r}-\zeta(t) \frac{2 \dot{R}(t)}{R(t)}=\beta D_{C} C_{r}-\frac{2 \alpha}{R(t)} \zeta(t) A_{r} \\
& =\beta D_{C} C_{r}-\frac{2 \alpha}{R(t)}(1-\zeta(t))\left(A-A^{*}\right) .
\end{aligned}
$$

Setting

$$
\eta(t)=\zeta(t)-1, \quad q(t)=\frac{2 \alpha}{R(t)}\left(A^{*}-A(R(t), t)\right.
$$

we can write

$$
\dot{\eta}+q(t) \eta=\beta D_{C} C_{r}(R(t), t) \equiv p(t)
$$


so that,

$$
\eta(t)=e^{-\int_{0}^{t} q(\tau) d \tau} \eta(0)+\int_{0}^{t} p(\tau) e^{-\int_{\tau}^{t} q(s) d s} d \tau
$$

Consider first the case that there exists a positive constant $\alpha_{0}$ and a sequence $t_{n} \rightarrow \infty$ such that

$$
\frac{1}{t_{n}} \int_{0}^{t_{n}}\left(A^{*}-A(R(\tau), \tau)\right) d \tau \geq \alpha_{0} \quad \text { as } \quad t_{n} \rightarrow \infty
$$

then,

$$
\int_{0}^{t_{n}} q(\tau) d \tau \geq \frac{2 \alpha}{R_{0}} \alpha_{0} t_{n} \quad \text { as } \quad t_{n} \rightarrow \infty
$$

By using (6.18) in (6.22), we get

$$
\begin{aligned}
\eta\left(t_{n}\right) & \geq \eta(0) e^{-\frac{2 \alpha \alpha_{0}}{R_{0}} t_{n}}+\int_{t_{0}}^{t_{n}} \frac{M}{\tau^{6}} e^{-\frac{2 \alpha A^{*}}{R_{*}}\left(t_{n}-\tau\right)} d \tau \\
& \geq \eta(0) e^{-\frac{2 \alpha \alpha_{0}}{R_{0}} t_{n}}+\frac{1}{2} \frac{R_{\star}}{2 \alpha A^{*}} \frac{M}{t_{n}^{6}}
\end{aligned}
$$

for some $t_{0}>0$ and all $t_{n}$ sufficiently large. This is a contradiction since $\eta(t)=\zeta(t)-1<0$ for all $t>0$.

It remains to consider the case where (6.23) is not satisfied for any $\alpha_{0}>0$, that is,

$$
\frac{1}{t} \int_{0}^{t} A(R(s), s) d s \rightarrow A^{*} \text { as } \quad t \rightarrow \infty .
$$

Since

$$
A_{r}(R(t), t)=\frac{1}{\alpha} \dot{R}(t) \rightarrow 0
$$

by Lemma 6.1 , we can derive, by comparison,

$$
A(r, t) \leq \widetilde{W}(r, t)
$$

for any $\varepsilon>0$ and $t>t_{\varepsilon}$, where $\widetilde{W}$ is the function introduced in (6.16). It follows that

$$
A(R(t), t) \rightarrow 0 \quad \text { if } \quad t \rightarrow \infty
$$

a contradiction to $(6.24)$. 
§7. Asymptotic estimates as $K \rightarrow \infty$. We now study the behavior of the solution $\left(A_{K}, B_{K}, C_{K}, R_{K}, \zeta_{K}\right)$ as $K \rightarrow \infty$, assuming that

$$
D_{B}=D_{A}
$$

Recall that

$$
0 \leq A_{K} \leq A^{*}, 0 \leq B_{K} \leq B^{*}
$$

and

$$
\frac{\partial}{\partial r} A_{K} \leq 0, \frac{\partial}{\partial r} B_{K} \geq 0, \dot{R}_{K}(t) \leq 0
$$

LEMMA 7.1. There exists a positive constant $M$ such that

$$
-M \leq \frac{\partial}{\partial r} A_{K}, \frac{\partial}{\partial r} B_{K} \leq M,-\alpha M \leq \dot{R}_{K}(t)
$$

for all $K$.

Proof. Consider the function $u=A_{K}-B_{K}$. It satisfies

$$
\begin{aligned}
& u_{t}=D_{A} u \quad \text { if } \quad r>R_{K}(t), t>0 \\
& -\zeta_{K, 0}(t) D_{A} u_{r}=\left(1-\zeta_{K, 0}(t)\right)\left(A^{*}-A\right) \text { if } r=R_{K}(t), t>0, \\
& u(r, 0)=A_{0}(r), r>R_{0}, \\
& u(\infty, t)=0, t>0
\end{aligned}
$$

where $\zeta_{K, 0}=\min \left\{\zeta_{K}, 1\right\}$. Applying the maximum principle to $u_{r}$ we deduce that

$$
-M \leq u_{r} \leq 0
$$

where $M$ is a constant independent of $K$. But then, upon recalling also (7.3),

$$
\left(A_{K, r}\right)^{2}=A_{K, r} u_{r}+A_{K, r} B_{K, r} \leq A_{K, r} u_{r} \leq M\left|A_{K, r}\right|
$$

so that $\left|A_{K, r}\right| \leq M$. The proof that $\left|B_{K, r}\right| \leq M$ is similar. Finally, $\dot{R}_{K}=\alpha A_{K, r} \leq-\alpha M$.

LEMma 7.2. There exist positive constants $N_{0}, N$ independent of $K$ such that, for any $T>0$,

$$
\int_{0}^{T} \int_{R_{K}(t)}^{\infty} K A_{K} B_{K} r^{2} d r d t \leq N_{0}+N T
$$

for all $K$.

Proof. Integrating the equation

$$
K A_{K} B_{K}=D_{A} \Delta A_{K}-\frac{\partial}{\partial t} A_{K}
$$


over $R_{K}(t)<r<\infty, 0<t<T$ and using Lemma 7.1, (7.7) readily follows.

In order to obtain uniform Hölder estimates on $A_{K}, B_{K}$, we consider the function $v=$ $A_{K} B_{K}$. It satisfies:

$$
\begin{gathered}
v_{t}=D_{A} \Delta v-2 A_{K, r} B_{K, r}-K\left(A_{K}+B_{K}\right) v, \quad r>R_{K}(t), \\
-\zeta_{K, 0}(t) D_{A} v_{r}=\left(1-\zeta_{K, 0}(t)\right)\left(A^{*}-A_{K}\right) B_{K}, r=R_{K}(t), \\
v(r, 0)=A_{0}(r) B_{0}(r), \quad r>R_{0}, \\
v(\infty, t)=0, t>0 .
\end{gathered}
$$

LEMma 7.3. For any $T>0$ and any compact set $\Omega_{T}$ in $\left\{R_{K}(t)<r<\infty, 0 \leq t \leq T\right\}$ whose distance to $r=R_{K}(t)$ is $\geq c_{*}>0$, there exists a constant $M$ depending on $T$ and $c_{*}$ (but not on $K$ ) such that

$$
\int_{\Omega_{T}}\left[K A_{K} B_{K}\left(A_{K}+B_{K}\right)\right]^{2} r^{2} d r d t \leq M
$$

Proof. Let $\xi(r, t)$ be a cutoff function such that $\xi=1$ in $\Omega_{T}$ and $\xi=0$ outside $\left(\frac{1}{2} c_{*}\right)$ neighborhood of $\Omega_{T}$. Multiplying the differential equation for $v$ by $\xi^{2} K v r^{2}$ and integrating, we obtain

$$
\begin{aligned}
& \int_{R_{K}(t)}^{\infty} \xi^{2} K \frac{v^{2}}{2} r^{2} d r+\int_{0}^{T} \int_{R_{K}(t)}^{\infty} \xi^{2} K\left(v_{r}\right)^{2} r^{2} d r d t \\
& \quad+\int_{0}^{T} \int_{R_{K}(t)}^{\infty} \xi^{2} K^{2} v^{2}\left(A_{K}+B_{K}\right) r^{2} d r d t \leq I
\end{aligned}
$$

where

$$
I=\int_{0}^{T} \int_{R_{K}(t)}^{\infty}\left[\left|\xi \xi_{t}\right| K v^{2}+2 \xi\left|\xi_{r}\right|\left|v_{r}\right| K v+2\left|A_{K, r} B_{K, r}\right| \xi^{2} K v\right] r^{2} d r d t
$$

is bounded independently of $K$, by Lemmas 7.1 and 7.2; this implies the assertion (7.10).

Lemma 7.4. Let $\Omega_{T}$ be as in Lemma 7.3. Then there exists a constant $M$ depending on $T$ and $c_{*}$ (but not on $K$ ) such that

$$
\begin{gathered}
\left\|A_{K}\right\|_{C^{1 / 4,1 / 8}\left(\Omega_{T}\right)} \leq M \\
\left\|B_{K}\right\|_{C^{1 / 2,1 / 8}\left(\Omega_{T}\right)} \leq M .
\end{gathered}
$$

That means that the $A_{K}$ and $B_{K}$ are uniformly Hölder continuous (in $\Omega_{T}$ ) with exponent $1 / 4$ in $r$ and exponent $1 / 8$ in $t$.

Proof. By Lemmas 7.2, 7.3,

$$
v_{t}-D_{A} \Delta v \text { is in } L^{2}\left(\Omega_{T}\right)
$$


uniformly in $K$. By $L^{2}$ estimates it then follows that

$$
\|v\|_{W_{2}^{2,1}\left(\Omega_{T}\right)} \leq M
$$

with a slightly smaller set $\Omega_{T}$ and a larger constant $M$, both independent however of $K$. By Sobolev's imbedding [11] we then have

$$
\|v\|_{C^{1 / 2,1 / 4}\left(\Omega_{T}\right)} \leq M
$$

with yet another constant $M$.

The function $u=A_{K}-B_{K}$ satisfies (7.5), and we can apply $L^{p}$ estimates to deduce $u$ also satisfies the estimate (7.13). Thus both $A_{K} B_{K}$ and $A_{K}-B_{K}$ belong to $C^{1 / 2,1 / 4}\left(\Omega_{T}\right)$, uniformly in $K$. Since

$$
A_{K}+B_{K}=\left(4 v+u^{2}\right)^{1 / 2}
$$

it follows that $A_{K}+B_{K}$ is in $C^{1 / 4,1 / 8}\left(\Omega_{T}\right)$, and the same then holds for $A_{K}$ and $B_{K}$.

THEOREM 7.5. Let $\widetilde{\Omega}_{T}$ be any compact which is contained in $R_{K}(t)<r<\infty, 0 \leq t<T$ for all $K$ sufficiently large. Then

$$
\lim _{K \rightarrow \infty} A_{K}(r, t) B_{K}(r, t)=0
$$

uniformly in $(r, t) \in \widetilde{\Omega}_{T}$.

Proof. By Lemma 7.2

$$
\iint_{\widetilde{\Omega}_{T}} A_{K} B_{K} r^{2} d r d t \leq \frac{M}{K} \rightarrow 0
$$

if $K \rightarrow \infty$. Since $A_{K} B_{K}$ is uniformly Hölder continuous in $\widetilde{\Omega}_{T}$ with exponent and coefficient independent of $K,(7.14)$ follows.

$\S 8$. Asymptotic limits as $K \rightarrow \infty$. The estimates of $\S 7$ show that for any sequence $K_{n}^{\prime} \rightarrow \infty$ there is a subsequence $K_{n}$ such that, as $K_{n} \rightarrow \infty$,

$$
\begin{aligned}
& R_{K_{n}}(t) \rightarrow R(t) \in \operatorname{Lip}[0, T] \text { in } C^{0}[0, T], \\
& A_{K_{n}}(r, t) \rightarrow A(r, t) \in C^{1 / 4,1 / 8}\left(Q_{T}\right) \text { in } C^{0}\left(Q_{T}\right), \\
& B_{K_{n}}(r, t) \rightarrow B(r, t) \in C^{1 / 4,1 / 8}\left(Q_{T}\right) \text { in } C^{0}\left(Q_{T}\right), \\
& K_{n} A_{K_{n}} B_{K_{n}} \rightarrow f \text { in the sense of weak convergence of measures, }
\end{aligned}
$$

for any $0<T<\infty$, where

$$
Q_{T}=\{(r, t) ; r>R(t), 0 \leq t<T\}
$$

and $f$ is a measure.

From Theorem 7.5 we have

$$
\begin{gathered}
A(r, t) B(r, t)=0 . \\
20
\end{gathered}
$$


The functions $A, B$ both satisfy the equation

$$
w_{t}-D_{A} \Delta w+f=0 \quad \text { in } \quad \mathcal{D}^{\prime}\left(Q_{T}\right),
$$

whereas the function $u=A-B$ satisfies

$$
u_{t}-D_{A} \Delta u=0 \quad \text { in } \quad Q_{T}
$$

since each of the functions $A_{K_{n}}-B_{K_{n}}$ satisfies this equation.

By (7.3) it follows that $u_{r} \leq 0$ in $Q_{T}$ and then, by the strong maximum principle,

$$
u_{r}<0 \text { in } Q_{T} \text {. }
$$

It follows that there exists a curve $r=S(t)$ with $S(t) \in C^{1+\nu}[0, T] \cap C^{\infty}(0, T)$ such that

$$
\begin{aligned}
& u(r, t)>0 \text { if } r<S(t), \\
& u(r, t)<0 \text { if } r>S(t) ;
\end{aligned}
$$

here $\nu$ is as in $(1.10),(1.11)$. Since $A_{0}-B_{0}$ is positive at $r=R_{0}$ and negative at $r=\infty$,

$$
R_{0}<S(0)<\infty
$$

Take $T$ such that

$$
S(t)>R(t) \text { for all } 0 \leq t \leq T
$$

For $r<S(t)$ we have $u>0$, or $A>B$. Since $A B=0$, it follows that $B=0$. Similarly $A=0$ if $r>S(t)$; thus

$$
A(r, t)=0 \quad \text { if } \quad r>S(t)
$$

$$
B(r, t)=0 \quad \text { if } \quad r<S(t)
$$

In any closed domain in $\{r<S(t), t<T\}$ we have $B=0$ and then, by (8.6) with $w=B, f=0$. Similarly $f=0$ if $r>S(t)$. It follows that $f$ is a measure supported on $r=S(t), 0<t<T$. In particular,

$$
A_{t}-D_{A} \Delta A=0 \quad \text { if } \quad r<S(t), \quad 0<t<T .
$$

Since also $A(S(t), t)=0$ and $S(t)$ is smooth, regularity results for the heat equation imply that $A$ is in $C^{1+\nu}$ in $R(t) \leq r \leq S(t), 0 \leq t \leq T$ and in $C^{\infty}$ in $R(t) \leq r \leq S(t), 0<t \leq T$.

Next, from (8.6) for $w=A$,

$$
-\iint A\left(-\varphi_{t}-D_{A} \Delta \varphi\right) r^{2} d r d t=\iint f \varphi r^{2} d r d t
$$

for any test function $\varphi$ in $Q_{T}$. Using the fact that $A=0$ if $r>S(t)$, and integrating by parts in (8.13) we find that

$$
\iint f \varphi r^{2} d r d t=-\int_{0}^{T} S^{2}(t) D_{A} A_{r}(S(t)-0, t) \varphi(S(t), t) d t
$$


It follows that

$$
f(r, t)=D_{A} A_{r}(S(t)-0,0) \delta(r-S(t)) .
$$

THEOREM 8.1. For any sequence $K_{n}^{\prime} \rightarrow 0$ there is a subsequence $K_{n} \rightarrow \infty$ such that the solutions of $\left(P_{K_{n}}\right)$ converge to a solution of $\left(P_{\infty}\right)$ uniformly in compact subsets of $\{r>$ $R(t), \quad 0 \leq t \leq T\}$

Proof. We have already proved most of the theorem. Since $S(t)>R(t)$ for $0 \leq t \leq T$, it follows that $A$ satisfies the two boundary conditions (2.8), (2.9), where $\zeta=\lim _{K \rightarrow \infty} \zeta_{K}$. Using the $C^{1+\mu}$-regularity of $A$ (which one obtains by the same argument as for Problem $\left(P_{K}\right)$ ) as well as the $C^{1+\mu}$-regularity of $R_{K}(t)$ and of the $A_{K}$ near $r=R_{K}(t)$, we can deduce that $C_{K_{n}} \rightarrow C$ uniformly near $r=R(t)$ and that $C$ satisfies the boundary condition (2.10). The remaining assertions of Theorem 8.1 have already been proved.

Denote by $T_{f}$ be the supremum of all $T$ 's for which (8.11) holds. We claim:

$$
\text { if } T_{f}<\infty \text { then } S(t) \rightarrow R\left(T_{f}\right) \text { if } t \rightarrow T_{f} .
$$

Indeed, if $\overline{\lim } S(t)>R\left(T_{f}\right)$, then (8.11) holds for $T>T_{f}$ (since $u$ is smooth and $u_{r}<0$ in $\{r>R(t), t>0\}$. On the other hand, if the limit $S\left(T_{f}-0\right)$ does not exist then $u_{r}\left(r, T_{f}\right)$ will vanish on a nonempty interval, contradicting the inequality $u_{r}<0$.

In $\S 9$ we shall prove uniqueness for Problem $\left(P_{\infty}\right)$; this implies that the convergence asserted in Theorem 8.1 is not just for a subsequence $K_{n} \rightarrow \infty$ but for all $K \rightarrow \infty$.

In $\S 10$ we shall prove that $T_{f}<\infty$, and this will conclude the proof of all the assertions made in Theorems 2.2 and 2.3 .

$\S 9$. Uniqueness for $\left(P_{\infty}\right)$. In this section we prove:

THEOREM 9.1. Assume that $D_{A}=D_{B}$. Then there exists at most one solution to Prob$\operatorname{lem}\left(P_{\infty}\right)$.

Proof. We begin with some remarks on the regularity of any solution $(A, B, C, R, S)$. Consider the function

$$
u=\left\{\begin{array}{rcc}
A & \text { if } & R(t) \leq r \leq S(t) \\
-B & \text { if } & r>S(t)
\end{array}\right.
$$

for all $t<T$, where $T<T_{f}$, the final time of the solution. Then

$$
u_{t}=D_{A} \Delta u
$$

and $u=A$ in a neighborhood of $\{(R(t), t), 0<t<T\}$. From this and the boundary conditions for $A$ and $C$ at $r=R(t)$ we easily deduce, as in earlier sections, that $R(t)$ and $\zeta(t)$ belong to $C^{1+\mu}[0, T]$ and $C(r, t)$ belongs to $C^{1+\mu}$ in $R(t) \leq r<S(t), \quad 0 \leq t \leq T$, for some $0<\mu<1$, and

$$
-M<u_{r}<0 \quad \text { for } \quad r>R(t), 0 \leq t \leq T \text {; }
$$

furthermore, the function $S(t)$ defined by

$$
r=S(t) \quad \text { if } u(r, t)=0
$$


is in $C^{1+\mu}[0, T]$ with

$$
\dot{S}(t)=-\frac{u_{t}(S(t), t)}{u_{r}(S(t), t)}
$$

(Note that $u_{t} \in C^{\mu}$ with $\mu=\nu$ up to $t=0$ since $A_{0}$ and $B_{0}$ belong to $C^{2+\nu}$ ). The function $C(r, t)$ is $C^{\infty}$ off $r=S(t)$. Near $r=S(t)$ the regularity of $C(r, t)$ is the same as the regularity of the special solution of (2.7):

$$
\widetilde{C}(|x|, t)=\int_{0}^{t} \int_{|y|=R(\tau)} \frac{e^{-\frac{|x-y|^{2}}{\sqrt{D_{C}} 4(t-\tau)}}}{\left(4 \pi^{2} D_{C}(t-\tau)\right)^{3 / 2}} u_{r}(S(\tau), \tau) d \sigma_{y} d \tau .
$$

This is a single layer potential and, since $u_{r}(S(\tau), \tau)$ is in $C^{\mu}$, this potential is in $C^{\beta}$ (across $r=S(t)$ ) for any $0<\beta<1$, and its derivative from each side of $r=S(t)$ is uniformly continuous (the proof is similar to the proof of Lemma 1 in $[6 ;$ p. 217]). Thus in particular,

$$
\left|C_{r}\right|_{L^{\infty}} \leq M^{\prime}, M^{\prime} \text { constant. }
$$

Suppose now that $\left(A_{i}, B_{i}, C_{i}, R_{i}, S_{i}\right)$ are solutions of Problem $\left(P_{\infty}\right)$ for $i=1,2$, and set

$$
u_{i}(r, t)=\left\{\begin{array}{lll}
A_{i}(r, t) & \text { if } & R_{i}(t) \leq r \leq S_{i}(t) \\
-B_{i}(r, t) & \text { if } & S_{i}(t)<r<\infty
\end{array}\right.
$$

for $0 \leq t \leq T$ where $T$ is such that

$$
S_{i}(t)-R_{i}(t) \geq \lambda>0 \text { for } 0 \leq t \leq T \text { and } i=1,2
$$

where $x=r-R_{i}(t)$, and

$$
\begin{aligned}
\tilde{u}(x, t) & =\widehat{u}_{1}(x, t)-\widehat{u}_{2}(x, t), \\
\widetilde{C}(x, t) & =\widehat{C}_{1}(x, t)-\widehat{C}_{2}(x, t) .
\end{aligned}
$$

Set

$$
\Omega_{\tau}=\{0<x<\infty, 0<t<\tau\} \quad \text { for any } \quad \tau \leq T \text {. }
$$

The functions $\widetilde{u}, \widetilde{C}$ satisfy (for simplicity we take $D_{A}=D_{C}=1$ ):

$$
\begin{aligned}
& \tilde{u}_{t}=\tilde{u}_{x x}+\left(\frac{2}{x+R_{1}}+\dot{R}_{1}\right) \tilde{u}_{x}+\left[\frac{2}{x+R_{1}}+\dot{R}_{1}-\frac{2}{x+R_{2}}-\dot{R}_{2}\right] \tilde{u}_{2, x} \text { in } \Omega_{T}, \\
& -\zeta_{1,0} \tilde{u}_{x}+\left(1-\zeta_{1,0}\right) \tilde{u}=\left(\zeta_{1,0}-\zeta_{2,0}\right)\left[\tilde{u}_{2, x}+\tilde{u}_{2}-A^{*}\right] \quad \text { if } \quad x=0,0<t<T, \\
& \tilde{u}(x, 0)=0, \quad x>0, \\
& \tilde{u}(\infty, t)=0, \quad 0<t<T,
\end{aligned}
$$


and

$$
\begin{aligned}
& \tilde{C}_{t}=\tilde{C}_{x x}+\left(\frac{2}{x+R_{1}}+\dot{R}_{1}\right) \tilde{C}_{x}+\left[\frac{2}{x+R_{1}}+\dot{R}_{1}-\frac{2}{x+R_{2}}-\dot{R}_{2}\right] \widehat{C}_{2, x} \\
& \quad+\widehat{u}_{2, x}\left(S_{2}-R_{2}, t\right) \delta\left(x+R_{2}-S_{2}\right)-\widehat{u}_{1, x}\left(S_{1}-R_{1}, t\right) \delta\left(x+R_{1}-S_{1}\right) \text { in } \Omega_{T}, \\
& -\tilde{C}_{x}+\gamma\left(\widehat{C}_{1}+\widehat{C}_{2}\right)\left(\widehat{C}_{1}^{2}+\widehat{C}_{2}^{2}\right) \tilde{C}=0 \text { if } x=0, \quad 0<t<T, \\
& \tilde{C}(x, 0)=0, \quad x>0, \\
& \tilde{C}(\infty, t)=0, \quad 0<t<T .
\end{aligned}
$$

By the maximum principle,

$$
\|\tilde{u}\|_{L^{\infty}\left(\Omega_{\tau}\right)} \leq\left\|\zeta_{1}-\zeta_{2}\right\|_{L^{\infty}(0, \tau)}+N \tau\left[\left\|R_{1}-R_{2}\right\|_{L^{\infty}(0, \tau)}+\left\|\dot{R}_{1}-\dot{R}_{2}\right\|_{L^{\infty}(0, \tau)}\right]
$$

for any $0<\tau<T$, where $N$ is a constant independent of $\tau$; in the sequel we shall denote by $N$ any such constant.

Differentiating in $x$ the differential equation in $(9.5)$, we obtain a parabolic equation for $\tilde{u}_{x}$. Using the maximum principle, we find that

$$
\left\|\tilde{u}_{x}\right\|_{L^{\infty}\left(\Omega_{\tau}\right)} \leq N\left\|\zeta_{1}-\zeta_{2}\right\|_{L^{\infty}\left(\Omega_{\tau}\right)}+N \tau\left[\left\|R_{1}-R_{2}\right\|_{L^{\infty}(0, \tau)}+\left\|\dot{R}_{1}-\dot{R}_{2}\right\|_{L^{\infty}(0, \tau)}\right] .
$$

Since $R_{1}(0)=R_{2}(0)$ we also have

$$
\left\|R_{1}-R_{2}\right\|_{L^{\infty}(0, \tau)} \leq \tau\left\|\dot{R}_{1}-\dot{R}_{2}\right\|_{L^{\infty}(0, \tau)} .
$$

From the boundary conditions for $\tilde{u}_{i}$ at $x=0$,

$$
\begin{aligned}
\left|\dot{R}_{1}-\dot{R}_{2}\right| & =\alpha\left|\widehat{u}_{1, x}(0, t)-\widehat{u}_{2, x}(0, t)\right| \\
& =\alpha\left|\frac{1-\zeta_{1,0}}{\zeta_{1,0}}\left(\widehat{u}_{1}-A^{*}\right)-\frac{1-\zeta_{2,0}}{\zeta_{2,0}}\left(\widehat{u}_{2}-A^{*}\right)\right|,
\end{aligned}
$$

so that

$$
\left\|\dot{R}_{1}-\dot{R}_{2}\right\| \leq N\left[\|\tilde{u}\|_{L^{\infty}\left(\Omega_{\tau}\right)}+\left\|\zeta_{1}-\zeta_{2}\right\|_{L^{\infty}(0, \tau)}\right] .
$$

Substituting (9.9), (9.10) into (9.7), (9.8) and choosing $\tau<1 /(2 N)$, we get

$$
\|\tilde{u}\|_{L^{\infty}\left(\Omega_{\tau}\right)}+\left\|\tilde{u}_{x}\right\|_{L^{\infty}\left(\Omega_{\tau}\right)} \leq N\left\|\zeta_{1}-\zeta_{2}\right\|_{L^{\infty}(0, \tau)} .
$$

Next, from the definition $(1.14),(1.15)$ of $\zeta_{i, 0}, \zeta_{i}$ we deduce that

$$
\left\|\zeta_{1}-\zeta_{2}\right\|_{L^{\infty}(0, \tau)} \leq N \tau\|\widetilde{C}(0, t)\|_{L^{\infty}(0, \tau)}+N\left\|R_{1}-R_{2}\right\|_{L^{\infty}(0, \tau)},
$$

and we need to estimate $\tilde{C}(0, t)$.

Denote by $G(x, y, t, s)$ the Green function for the problem (see [3])

$$
\begin{aligned}
& w_{t}=w_{x x}+\left(\frac{2}{x+R_{1}}+\dot{R}_{1}\right) w_{x} \text { in } \Omega_{T}, \\
& -w_{x}+\gamma\left(\widehat{C}_{1}+\widehat{C}_{2}\right)\left(\widehat{C}_{1}^{2}+\widehat{C}_{2}^{2}\right) w=0 \text { if } x=0, \quad 0<t<T, \\
& w(\infty, t)=0, \quad 0<t<T .
\end{aligned}
$$


Then (using $(9.6))$ we can represent $\tilde{C}$ in the form

$$
\begin{aligned}
& \tilde{C}(|x|, t)=\int_{0}^{t} \int_{0}^{\infty} G(x, y, t, s)\left[\frac{2}{y+R_{1}(s)}+\dot{R}_{1}(s)-\frac{2}{y+R_{2}(s)}-\dot{R}_{2}(s)\right] \hat{C}_{2, y}(y, s) d y d s \\
& \quad+\int_{0}^{t} G\left(x, S_{2}(s)-R_{2}(s), t, s\right) \widehat{u}_{2, x}\left(S_{2}(s)-R_{2}(s), s\right) d s \\
& \quad-\int_{0}^{t} G\left(x, S_{1}(s)-R_{1}(s), t, s\right) \widehat{u}_{1, x}\left(S_{1}(s)-R_{1}(s), t-s\right) d s . \\
& \equiv J_{1}+J_{2}+J_{3} .
\end{aligned}
$$

We can write

$$
\begin{aligned}
& \left|J_{2}(0, t)+J_{3}(0, t)\right| \leq \int_{0}^{t}\left|G\left(0, S_{2}-R_{2}, t, s\right)\right|\left|\widehat{u}_{2, x}\left(S_{2}-R_{2}, s\right)-\widehat{u}_{1, x}\left(S_{1}-R_{1}, s\right)\right| \\
& \quad+\int_{0}^{t}\left|\widehat{u}_{1, x}\left(S_{1}-R_{1}, s\right)\right|\left|G\left(0, S_{2}-R_{2}, t, s\right)-G\left(0, S_{1}-R_{1}, t, s\right)\right|
\end{aligned}
$$

where $S_{i}=S_{i}(s), R_{i}=R_{i}(s)$. The difference in the first integral is equal to

$$
\left[\widehat{u}_{2, x}\left(S_{2}-R_{2}, s\right)-\widehat{u}_{2, x}\left(S_{1}-R_{1}, s\right)\right]+\tilde{u}_{x}\left(S_{1}-R_{1}, s\right) .
$$

Therefore, if $0<t<\tau$,

$$
\left|J_{2}(0, t)-J_{3}(0, t)\right| \leq N\left[\left\|S_{1}-S_{2}\right\|_{L^{\infty}(0, \tau)}+\left\|R_{1}-R_{2}\right\|_{L^{\infty}(0, \tau)}+\left\|\tilde{u}_{x}\right\|_{L^{\infty}\left(\Omega_{\tau}\right)}\right] .
$$

Using the regularity result (9.3), we can immediately estimate also $\left|J_{1}(0, t)\right|$ by the $L^{\infty}$ norm of $\left|R_{1}-R_{2}\right|+\left|\dot{R}_{1}-\dot{R}_{2}\right|$. Hence

$$
|\tilde{C}(0, t)| \leq N\left[\left\|\dot{R}_{1}-\dot{R}_{2}\right\|_{L^{\infty}(0, \tau)}+\left\|S_{1}-S_{2}\right\|_{L^{\infty}(0, \tau)}+\left\|\tilde{u}_{x}\right\|_{L^{\infty}\left(\Omega_{\tau}\right)}\right] .
$$

Next we need to estimate $S_{1}-S_{2}$. By the mean value theorem,

$$
\widehat{u}_{1}\left(S_{1}-R_{1}, t\right)-\widehat{u}_{1}\left(S_{2}-R_{2}, t\right)=\widehat{u}_{1, x}(y, t) \cdot\left(S_{1}-R_{1}-S_{2}+R_{2}\right)
$$

where $y$ is a point between $S_{1}-R_{1}$ and $S_{2}-R_{2}$. Since $\left|u_{1, x}\right| \geq \nu_{0}>0\left(\nu_{0}\right.$ constant which depends on the $\lambda$ in (9.4)), we get

$$
\begin{aligned}
& \left|S_{1}(t)-S_{2}(t)\right| \leq\left|R_{1}-R_{2}\right|+\frac{1}{\nu_{0}}\left|\widehat{u}_{1}\left(S_{1}-R_{1}, t\right)-\widehat{u}_{1}\left(S_{2}-R_{2}, t\right)\right| \\
& =\left|R_{1}-R_{2}\right|+\frac{1}{\nu_{0}}\left|\widehat{u}_{2}\left(S_{2}-R_{2}, t\right)-\widehat{u}_{1}\left(S_{2}-R_{2}, t\right)\right| \\
& \leq|| R_{1}-R_{2} \|_{L^{\infty}(0, \tau)}+\frac{1}{\nu_{0}} \underset{25}{\|\tilde{u}\|_{L^{\infty}\left(\Omega_{\tau}\right)} .}
\end{aligned}
$$


Substituting this into (9.13) and using the result in (9.12), we find after using also (9.10) and (9.11), that $(9.12)$ becomes

$$
\left\|\zeta_{1}-\zeta_{2}\right\|_{L^{\infty}(0, \tau)} \leq N \tau\left\|\zeta_{1}-\zeta_{2}\right\|_{L^{\infty}(0, \tau)} .
$$

Hence $\zeta_{1}(t)=\zeta_{2}(t)$ if $0 \leq t \leq \tau, \tau$ small. This implies (by (9.11) and (9.10)) that $u_{1} \equiv$ $u_{2}, \quad R_{1} \equiv R_{2}$ and the two solutions coincide if $0 \leq t \leq \tau$. We similarly proceed step-by-step to complete the proof of uniqueness.

$\S 10 . T_{f}<\infty$.

In this section we prove:

THEOREM 10.1. If $D_{A}=D_{B}$ and $C_{0}(r) \not \equiv 0$ then $T_{f}<\infty$.

We shall denote by $T_{\infty}^{*}$ the smallest $t$ (if existing) such that $\zeta(t) \geq 1$, and first prove:

LEMMA 10.2. If $T_{f}=\infty$ then $T_{\infty}^{*}<\infty$.

Proof. Suppose the assertion is not true; then $\zeta(t)<1$ for all $t \geq 0$. Since also $T_{f}=$ $\infty, \quad A(r, t)=u(r, t)$ for all $(r, t)$ in a neighborhood of $\{(R(t), t), 0 \leq t<\infty\}$ and, consequently,

$$
\begin{gathered}
D_{A} \zeta u_{r}+(1-\zeta)\left(u-A^{*}\right)=0 \quad \text { if } \quad r=R(t), t>0, \\
u_{r}=\alpha \dot{R} \quad \text { if } \quad r=R(t), t>0 .
\end{gathered}
$$

where $u=\lim _{K \rightarrow \infty}\left(A_{K}-B_{K}\right)$. Set

$$
\sigma=\lim _{t \rightarrow \infty} \zeta(t) ; \text { then } \sigma \leq 1
$$

If $\sigma<1$ then we can use the comparison argument as in the proof of $(6.7)$ to deduce that

$$
\left|u(r, t)-u_{\infty}(r)\right| \leq \widetilde{W}(r, t) \text { for all } t>0,
$$

where $\widetilde{W}$ is defined in (6.16) and $u_{\infty}(r)$ is the harmonic function in $\rho<r<\infty$ where $(\rho=$ $\left.\lim _{t \rightarrow \infty} R(t)\right)$ satisfying

$$
-D_{A} \sigma u_{\infty, r}(\rho)+(1-\sigma)\left(u_{\infty}(\rho)-A^{*}\right)=0, u_{\infty}(\infty)=-B^{*} .
$$

Next we argue as in Lemma 6.2 (with $t_{0}=0$ in (6.19)) and deduce that

$$
C_{K, r}\left(R_{K}(t), t\right) \geq \frac{M}{t^{6}} \quad(M>0, \quad t \geq 1) .
$$

where $M$ is independent of $K$. Hence

$$
C_{r}(R(t), t) \geq \frac{M}{t^{6}} \quad(M>0, \quad t \geq 1) .
$$

Using this estimate and (10.1), (10.2), we can proceed as in the proof of Theorem 6.3 , deriving $(6.22)$ for $\eta(t)=\zeta(t)-1$, and concluding that if

$$
\frac{1}{t_{n}} \int_{0}^{t_{n}}\left(A^{*}-u(R(\tau), \tau)\right) d \tau \geq \alpha_{0}>0
$$


for a sequence $t_{n} \rightarrow \infty$, then $\eta\left(t_{n}\right)>0$ for $t_{n}$ large enough, which is a contradiction to the assumption that $\zeta(t)<1$ for all $t>0$. Hence

$$
\frac{1}{t} \int_{0}^{t} u(R(\tau), \tau) d \tau \rightarrow A^{*} \quad \text { as } \quad t \rightarrow \infty .
$$

This together with (10.3) implies that $u_{\infty}(\rho)=A^{*}$. Hence $u_{\infty}$ takes it maximum at $r=\rho$ and, by the maximum principle, $u_{\infty, r}(\rho)<0$. However, since $u_{\infty}(\rho)=A^{*}$, we must also have $u_{\infty, r}(\rho)=0$ by (10.4), which is a contradiction. We conclude that $\sigma=1$, i.e., $\zeta(\infty)=1$ and then, by (10.1),

$$
u_{r}(R(t), t) \rightarrow 0 \quad \text { if } t \rightarrow \infty
$$

By (10.9) and a comparison argument (as in the proof of Lemma 6.1) we then get

$$
u(r, t) \leq \widetilde{W}(r, t)
$$

where $\widetilde{W}$ is defined in $(6.16)$; therefore

$$
u(R(t), t) \rightarrow 0 \quad \text { if } \quad t \rightarrow \infty
$$

Observe next that the argument that led from (10.5) to (10.8) is independent of whether $\sigma<1$ or $\sigma=1$. Thus (10.8) still holds and this is a contradiction to (10.10). The proof that $T_{\infty}^{*}$ is finite is thereby complete.

Proof of Theorem 10.1. Suppose $T_{f}=\infty$. Then, by Lemma 10.2, $T_{\infty}^{*}<\infty$. Therefore

$$
u_{r}(\bar{R}, t)=0 \quad \text { if } \quad t>T_{\infty}^{*}\left(\bar{R}=R\left(T_{\infty}^{*}\right)\right) .
$$

Since also $u_{t}=D_{A} \Delta u$ and $u(\infty, t)=-B^{*}$, it follows by the comparison argument that

$$
\left|u(r, t)+B^{*}\right| \leq M \frac{e^{-\frac{r^{2}}{\sqrt{D_{A}} 4 t}}}{t^{3 / 2}} \quad \text { if } \quad t>T_{\infty}^{*} .
$$

Consequently, $u(r, t)<0$ for all $r \geq R(t), t \geq T$ provided $T$ is sufficiently large, and this is a contradiction to the assumption that $T_{f}=\infty$ (which implies that $u$ remains positive for all $t>0)$.

Acknowledgement. The first author is partially supported by National Science Foundation Grant DMS87-22187.

\section{REFERENCES}

[1] G. Astasita, Mass Transfer with Chemical Reaction, Elsevier, Amsterdam, 1967.

[2] J.R. Cannon and C.D. Hill, On the movement of a chemical reaction interface, Indiana Univ. Math. J., $20(1970), 429-454$.

[3] S.D. Eidelman, Parabolic Systems, North-Holland, Groningen, 1969.

[4] P. Erdi and J. Toth, Mathematical Models of Chemical Reactions, Princeton University Press, Princeton, N.J., 1989.

[5] L.C. Evans, A convergence theorem for a chemical diffusion-reaction system, Houston J. Math., 6 (1980), $259-267$. 
[6] A. Friedman, Partial Differential Equations of Parabolic Type, Prentice-Hall, Englewood Cliffs, N.J., 1964.

[7] A. Friedman, One phase moving boundary problems, Conference on Moving Boundary Problems, Academic Press, New York, 1978, 25-40.

[8] A. Friedman, Variational Principles and Free-Boundary Problems, Wiley-Interscience, New York, 1982.

[9] A. Friedman, Mathematics in Industrial Problems, Part 6, IMA Volume 57, Springer-Verlag, New York, 1993.

[10] D. Kinderlehrer and G. Stampacchia, An Introduction to Variational Inequalities and Their Applications, Academic Press, New York, 1986.

[11] O.A. Ladyzhenskaja, V.A. Solonnikov and N.N. Uraltseva, Linear and Quasilinear Equations of Parabolic Type, Amer. Math. Soc. Transl., American Mathematical Society, Providence, R.I., 1968.

[12] R.H. Martin Jr., Mathematical models in gas-liquid reactions, Nonlinear Analysis, 4 (1980), 509-527.

[13] A.E. Nielsen, Kinetics of Precipitation, Pergamon Press, Oxford, 1964.

[14] A. Visintin, Stefan problem with a kinetic condition at the free boundary, Ann. Math. Pura Appl., 146 (1987), 97-122.

[15] W. Xie, The Stefan problem with a kinetic condition at the free boundary, SIAM J. Math. Anal., 21 (1990), 362-373. 